\title{
An adolescent's subjective experiences of mindfulness
}

\section{Carey-Ann Dellbridge and Carien Lubbe*}

\author{
Department of Educational Psychology, Faculty of Education, All Purpose Building C114, \\ Groenkloof Campus, University of Pretoria, Pretoria 0002, South Africa \\ *Corresponding author, email: carien.lubbe@up.ac.za
}

\begin{abstract}
An adolescent's subjective experiences of mindfulness were explored in a single case study of a 17-yearold female. Data were created by means of 'mindfulness sessions', unstructured interviews, creative expression, journals and field notes. The data were analysed and interpreted using a combination of typological and interpretive analysis strategies. Findings are presented within a conceptual framework of mindfulness derived by the main author from the literature review. Five themes emerged from the data in relation to the conceptual framework applied, namely present-centered awareness and attention, attitude and heart qualities, self-regulation, universalism and mindlessness. Overall findings suggest firstly that the participant subjectively experienced mindfulness as being predominantly task-oriented. Secondly, it appears that the participant experienced personal growth and development in terms of her understanding and practice of mindfulness. These findings could make a potential contribution towards qualitative research on mindfulness, and research on how mindfulness could possibly apply to an adolescent. Studies have shown mindfulness to be a potentially promising intervention and quality to be cultivated in the development of well-being. The study is thus significant in the context of positive psychology and a move towards more holistic health and well-being.
\end{abstract}

\section{Introduction}

The focus of this paper is to report on a case study that explored an adolescent's subjective experiences of mindfulness. The concept of mindfulness has become prominent in the field of psychology in recent years, especially from a positive psychological framework (Brown and Ryan 2003, Brown, Ryan and Creswell 2007, Siegel 2007). Psychological research into mindfulness has focused primarily on the effects of training, usually as part of a clinical treatment package, and less so on understanding the meaning and expression of mindfulness itself (Brown et al. 2007). According to professional knowledge, limited research has been done on how clients themselves experience mindfulness as an intervention strategy or therapeutic technique. More importantly, there is limited research into the applicability of mindfulness to children, adolescents and the youth, with most studies having focused on adult populations (Baer 2003, Matchim and Armer 2007).

Given its potential promise as identified across a range of contexts, mindfulness may be a valuable asset to be applied within adolescence. This is especially relevant in the context of the current international focus on positive psychology, which aims to "create a science of human strength whose mission will be to understand and learn how to foster these virtues in young people' (Seligman and Csikzentmihalyi 2000: 7). Furthermore, it appears that a holistic approach to 'healing', therapy, health and well-being, focusing on the integration of mind, body and soul, is currently infiltrating the field of psychology. Gaining insight into how an adolescent experiences mindfulness may generate further understanding or research into the applicability of mindfulness to 
an important section of the population, within the context of current trends and movements towards a positive psychology and integrated holistic approaches to health and well-being (Coetzee and Viviers 2007).

Therefore, the primary aim of the study was to generate a detailed understanding of an adolescent's subjective experiences of mindfulness. Secondly, the study aimed to gain insight regarding how, and if, an adolescent might perceive mindfulness, make sense of it, conceptualise it, and possibly relate to it as a potential asset to be incorporated into his or her life, or not. A further aim was thus to establish whether mindfulness 'makes sense' to an adolescent, given this specific developmental context, and whether or not the adolescent could possibly embrace it as a workable technique. In light of the rationale and purpose of the study as described above, the research question was posed as follows: How does an adolescent subjectively experience mindfulness? This paper provides an overall synthesis of the findings of this study.

\section{Exploring mindfulness}

It is evident that there are various perspectives and approaches to the complex phenomenon of mindfulness, which appears to be, paradoxically, both an age-old and modern concept. Germer (2005) distinguishes between three phenomena that the word mindfulness can be used to describe; namely, a theoretical construct (mindfulness), a practice of cultivating mindfulness (such as meditation), or a psychological process (being mindful). In examining mindfulness, this paper makes reference to all three terms, and these are used interchangeably, depending on the context of the discussion.

Mindfulness can mostly be associated with eastern meditation practices. However, it is evident that the concept also exists within Western psychological origins, such as in the Gestalt approach. Dr Jon Kabat-Zinn could be regarded as one of the modern forerunners of this movement, with his development of the Mindfulness-Based Stress Reduction (MBSR) programme in 1979. This study adopted Kabat-Zinn's operational working definition of mindfulness as, 'the awareness that emerges through paying attention on purpose, in the present moment, and non-judgmentally to the unfolding of experience moment by moment' (Kabat-Zinn 2003: 145). Ideally, mindful behaviour implies that an individual is fully 'in contact' with what is taking place in the present moment, including the external reality as well as his or her internal responses to it (Thompson and Gauntlett-Gilbert 2008).

Thompson and Gauntlett-Gilbert (2008) describe an example of how mindfulness could be ideally applied. For instance, if one were walking to school in a 'mindful' way, one would be aware of physical sensations such as one's feet hitting the floor and the weight of a school bag on one's shoulder. One would also be aware of internal experiences; for example, the mild tension associated with approaching the school gates. One of the goals of mindfulness is that one would focus on this awareness, instead of giving attention to other phenomenona such as future exams or party one attended the night before. If one's mind did wander, however, one would observe the wandering non-judgmentally, and gently bring one's awareness back to the present moment.

Mindfulness has potential promise as a quality to be cultivated. It is a way of relating to all human experiences, whether positive or negative, which allows one to be less reactive and therefore less vulnerable to negative states. In this way, mindfulness may reduce our overall suffering and a sense of well-being may increase (Germer 2005). A greater non-judgemental awareness of one's own impulses and thought patterns thus results in decreased emotional reactivity and vulnerability. When individuals deliberately stay in the present moment, they are more able to respond to events with awareness of their automatic tendencies, and to make choices that are not necessarily negatively influenced by these (Thompson and Gauntlett-Gilbert 2008).

Following an in-depth analysis of the literature pertaining to mindfulness, five themes emerged that serve to illustrate the various layers of this complex phenomenon, which, on the surface appears deceptively simple. These themes were extracted from the review and adopted as the conceptual framework applied in this study. They are referred to as the 'dimensions' of mindfulness, namely: present-centred awareness and attention, attitude and heart qualities, self-regulation, universalism and mindlessness. These dimensions are elaborated on and discussed in the findings section. 


\section{Research with children and adolescents}

Despite the large gap in the literature pertaining to mindfulness-based interventions with children and adolescents, there are a few studies to indicate its use within these populations. Most research points to the potential beneficial results that young people can attain from participating in mindfulness techniques; for example: increased positive psychological states and traits that result from mindfulness practice, and reductions in negative states such as anxiety and aggression.

It has been suggested that mindfulness practices are essentially attention-enhancing techniques. Therefore, since impaired attention is a core symptom of anxiety, enhancing self-management of attention would reduce anxiety in children (Semple, Reid and Miller 2005). Mindfulness has also been found to modulate aggression in adolescents. Research indicates that adolescents were able to learn a mindfulness technique and could successfully apply it in situations that had previously triggered aggressive responses (Singh et al. 2007). Singh et al. (2007) list the benefits of the technique: it is simple to learn; can be applied in all settings, including negative and high-arousal situations, and it has been proven effective in the self-control of aggressive behaviour.

With regards to the positive outcomes of mindfulness practice by children, it has been suggested that children participating in a five-week programme combining Tai Chi with the MindfulnessBased Stress Reduction (MBSR) programme experienced a range of benefits; including well-being, calmness, relaxation, improved sleep, less reactivity, greater self-care and self-awareness, and a sense of interconnection or interdependence with nature (Wall 2005). Adolescents who have received treatment for substance abuse have also indicated improved sleep as a result of participation in a treatment programme including MBSR as one of its components (Bootzin and Stevens 2005).

Thompson and Gauntlett-Gilbert (2008) assert that, despite the lack of empirical evidence in this domain, there is considerable alternative evidence that mindfulness is potentially teachable and beneficial to child and adolescent populations. This is the identified gap in which the study potentially fits. It was envisaged that by developing in-depth understandings of an adolescent's subjective experiences of mindfulness, this may ultimately contribute towards our insight into how mindfulness can be applied within this population group.

\section{Method}

An interpretivist paradigm facilitated the process of generating an in-depth understanding of an adolescent's subjective experiences of mindfulness, as she has created and given them meaning within her personal reality. An interpretivist stance towards reality implies that multiple realities are possible, as experienced by different individuals. Individuals' experiences of reality are ultimately shaped by their constructions of beliefs and meanings as they interact with their world. The interpretivist paradigm thus also informed the methodological approaches applied in this study - that of the qualitative tradition. Qualitative research methods enable the rich and detailed descriptions necessary to develop understanding of an individual's experiences.

\section{Research design}

The study was conducted using an intrinsic case study design in which the bounded system was a single case of an adolescent in her life context, within a specific time frame (Stake 2005, Yin 2003). The case study method was applied in order to facilitate the in-depth exploration required to develop a comprehensive understanding of the case and contexts. It enabled the study of a unique example of a real-life person and situation, and her subjective experiences. It facilitated an in-depth investigation that appreciates the 'wholeness' of a person and situations, and allowed for rich descriptions. The case study design allows readers to develop more in-depth understandings, and is more accessible to wider audiences. This is particularly useful in the study, as from the literature review it was evident that detailed and in-depth understandings of peoples' experiences of mindfulness appear to be few and far between. A case study design is able to tie together theory and practice. The study examined an adolescent's subjective experiences of mindfulness within the context of theory, and thus facilitated an understanding of how the theory of mindfulness might relate to an 
adolescent in real-life practice. Furthermore, a case study can provide insight into other similar situations, and it is possible that the generalisation of results can be made to theory - advantages consistent with aspects of the purpose and rationale of this study.

\section{Participant}

The participant in the study was purposefully selected according to predetermined criteria, which were based on the research topic. Selection criteria included the following aspects. The participant had to be an adolescent with the ability for metacognition and reflexive thought processes. Superior language ability was taken into account, as well as physical proximity to the researcher and availability. The purposeful sampling method was the most appropriate method as it allowed for the selection of a participant who could best facilitate a thorough exploration of the research topic. The participant will be referred to as Lia (a pseudonym). She is 17 years of age and at the time of study was in her final school year.

\section{Data gathering}

The data in this study were created through several avenues. 'Mindfulness sessions' were conducted between the researcher and participant, by means of participant observation, where both took part in various mindfulness activities. These five mindfulness sessions were conducted between the researcher and participant over a period of ten weeks. Both direct and indirect mindfulness training techniques were employed during the sessions. Direct techniques included an initial explanation of what mindfulness is in theory, as well as the researcher's own interpretations and experiences of it during the discussions. The audio CD 'Mindfulness for Beginners' of Dr Jon Kabat-Zinn (Kabat-Zinn 2006) was used during the sessions. On the CD Kabat-Zinn discusses the principles of mindfulness and guides the listener through several meditations which form the basis of his Mindfulness-Based Stress Reduction (MBSR) programme. Furthermore, unstructured interviews were held, which facilitated the development of the in-depth understandings of the case, as required by the nature of the research. Data were also created from several forms of 'creative expression' (i.e. artwork) that were created by the participant during the mindfulness sessions, as well as from the participant's research journal. All interviews were transcribed, resulting in the transcriptions that formed a large part of the study's data. Data also included field notes, which consisted of the researcher's personal research journal and observations made during the study.

\section{Data analysis and interpretation}

The data analysis and interpretation stage of the study involved a combination of typological and interpretive analysis. A preliminary exploratory analysis was conducted first (Creswell 2002). This entailed carefully reading through all the transcripts to obtain a general sense of them, making notes of initial comments and ideas, thinking about the organisation of the data, and considering whether more data were needed (Creswell 2002, Hatch 2002). Next the transcripts, journal entries and visual data from each session were focused on, in chronological order. This was also part of the first step - getting a sense of the whole; but in this case the whole was the session itself. A mind map was then created indicating the main topics covered during the session, as well as impressions of emerging themes.

Typological analysis began by dividing the data into categories based on predetermined typologies. The typologies were generated from theory, and were the dimensions that emerged from the research into the theory on mindfulness. Therefore 'present-centred awareness and attention', 'self-regulation', 'attitude and heart qualities', 'universalism', and 'mindlessness' were identified as the five typologies to work with. The following step in the typological analysis was to read all the transcripts, field notes and journal entries, marking entries that related to each category. One typology was focused on at a time, with a particular colour representing it. Separate files were then created for each typology. Therefore, all the data entries related to 'self-regulation', for instance, were extracted and put together into one document, creating five new files containing all the data entries related to individual typologies. 
Once again, the data were read for a sense of the whole, except this time the whole constituted all the data within each typology. At this point the interpretive aspect of the data analysis became more salient as we started to look for meaning within the data from each typology. Underlying implications were identified and similar and reoccurring topics were clustered. Codes were devised according to initial interpretations of the data and the search for meanings. Codes were developed for each topic that was identified in the previous step, and then the authors systematically went through the typology files, writing the descriptive codes next to each section of data that they matched. This process helped to develop and clarify the themes that emerged. By examining the coded data, it was possible to confirm dominant themes, including searching the data for examples that contradicted the identified themes.

An appropriate descriptive label was devised for each theme, and we created a mind map indicating the themes around each typology, as well as more detailed notes on interpretations of the data within each theme. The last independent step of data analysis and interpretation was to review all the original transcripts, the typology files, journal, field notes and artwork, to ensure that the findings and themes were consistent with the data, and last but not least, the first author engaged in 'member checking' (Lincoln and Guba 1985) - a vital part of data analysis and interpretation in qualitative research.

\section{Quality criteria and ethical considerations}

Adhering to certain quality criteria developed trustworthiness of the study. Credibility (De Vos et al. 2005) was established by defining the boundaries and parameters of the study. Furthermore, the qualitative methodological technique of crystallisation (Denzin and Lincoln 2003) contributed towards credibility. The quality criteria of transferability and dependability (Lincoln and Guba 1985, Yin 2003) were also addressed. The ethical considerations of informed consent, safety in participation, trust, confidentiality, and credibility of the researcher were also taken into account for the duration of the study. A large part of the data creation process of the study involved the use of CD entitled 'Mindfulness for Beginners' by Jon Kabat-Zinn (Kabat-Zinn 2006). Permission to make use of this material was obtained from the participant prior to commencement of the study.

\section{Results}

The conceptual framework consisting of the five dimensions (present-centred attention and awareness, attitude and heart qualities, self-regulation, universalism of mindfulness, and mindlessness) were applied as a lens through which to analyse and interpret the data in this study. Within each dimension, several themes emerged from the data that serve to illustrate the apparent nature and details of Lia's mindfulness experiences (see Figure 1). The findings of the study are differentiated in terms of primary and secondary themes. It seems that Lia experienced primary themes within the first three dimensions of mindfulness (present-centered attention and awareness, attitude and heart qualities, and self-regulation), with the last two dimensions (universalism of mindfulness and mindlessness) indicating secondary themes experienced. Although these are presented one at a time, as mentioned, it is important to note that the dimensions of mindfulness are closely interlinked, and come together in a dynamic relationship to create the full experience of mindfulness.

\section{Primary theme 1: Present-centred attention and awareness}

The core of mindfulness relates to aspects of human consciousness, specifically, attention and awareness. According to Brown and Ryan (2003: 822) awareness is the 'background radar' of consciousness, and attention is a process of focusing awareness on something specific. For example, we may drive a familiar road 'on autopilot', being vaguely aware of the road, but our attention could be focused on a child running across the road or perhaps an accident up ahead (Germer 2005). Thus our attention continuously pulls 'figures' out of the 'ground' of awareness, and holds that focus for varied lengths of time.

Lia's present-centred attention and awareness in mindfulness seems to be predominantly task-oriented, resulting in her focusing on attention more than awareness. That is, she identified a 
Figure 1: Lia's subjective experiences located within the dimensions of mindfulness

\begin{tabular}{lcc}
\hline & Primary themes & \\
\hline $\begin{array}{l}\text { Present-centred attention and } \\
\text { awareness }\end{array}$ & Attitude and heart qualities & Self-regulation \\
\hline Task-orientated & Intention & Interest-driven \\
External vs. Internal awareness & Perfectionism & Needs silence \\
Enhanced sensory experience & Letting go & Requires effort \\
& & Improves with awareness \\
\hline & Secondary themes & \\
\hline $\begin{array}{l}\text { Universalism } \\
\text { Science and religion } \\
\text { Everyday life }\end{array}$ & Awareness of mindlessness \\
\hline
\end{tabular}

task to direct her attention towards in the present moment, and appeared to interpret mindfulness as the successful focused attention on present tasks:

'I can hear the cars and the crickets and those children playing but I'm still trying to focus on what you're saying.'

On the whole, Lia's specific experience in this regard appears to be unrelated to what the theoretical literature implies. The study suggested that Lia experienced mindfulness as directing her attention to a specific task in the present, as opposed to directing both attention and awareness to the general unfolding of experiences, as they occur in each moment. Lia's experience was to engage in interpretations of what was important to direct her attention towards, as well as interpretations of present experiences. It appears that for the most part, she did not experience mindfulness as more 'neutral' and focused predominantly on the 'quality of consciousness itself' (Brown and Ryan 2003). By being task-oriented, Lia was concerned about what her attention should or should not be focused on, and in this process tended to favour present-centred attention over awareness.

In addition, her task-oriented attention appeared to interfere with a fundamental clarity of awareness, and possibly prevented her from experiencing mindfulness as a 'polished mirror', which simply reflects what passes before it, unbiased by conceptual thoughts about what is happening at the given moment (Brown et al. 2007). That is, Lia seemingly tended to involve her attention in more interpretations of her present experiences, instead of observation alone. This noted, however, it is crucial to point out the extreme difficulty of attaining such a 'perfect' state of mindful awareness. Nowhere in the literature is it implied that this is an easy state to achieve; rather, it is clear that mindfulness practice is exactly that - practise! As Kabat-Zinn (2005) explains, even the masters of mindfulness meditation will claim that they know nothing about the subject after seemingly endless hours and years of mindfulness practice. This brings to light one of the limitations of this study and something that must be kept in mind while examining the findings; the concept of mindfulness was completely new to Lia and she had never practiced it before. Furthermore, her exposure to mindfulness was limited to our discussions, which included the authors' personal interpretations, Kabat-Zinn's CD, and her own 'experimentation' with guided and non-guided mindfulness activities. Thus the study can almost be viewed as an initial 'experimentation' with mindfulness for Lia, and her subjective experiences thereof are described, bearing in mind the identified limitations of this particular context.

The second theme emerging from the 'present-centred attention and awareness' dimension suggests that Lia's present-centred awareness included more external happenings than internal ones. She thus experienced this dimension of mindfulness as being more aware of external phenomena such as sounds, as opposed to internal experiences such as thoughts or feelings: 
watching TV I wouldn't be aware of, oh there's children playing outside, or there's birds like chirping or whatever, or there's a wind blowing, I would just be watching TV and also not really grasping what l'm watching on TV. So now l'm aware of everything else as well.'

'... it was wandering [her mind] because you can still hear the distractions outside, so I found myself at times focusing more on that than I was with the meditation.'

These statements highlight the relationship between attention and awareness in mindfulness practice. These two phenomena are interlinked, in that attention is really a focused awareness; attention is conscious awareness focused on a particular thing for a certain amount of time. True mindfulness practice requires a flexible flow between attention and awareness in the present moment. In this example, Lia had identified the meditation as the task to focus her present-centred attention on. She demonstrated mindfulness in that she became aware of external sounds in the environment and then moved her focused attention to them. However, she interpreted these sounds as distractions that prevented her full attention being given to the meditation task. Once again, the interpretations Lia made of her present experiences illustrate the difference between her own experiences of mindfulness and those described in the literature, which emphasise refraining from interpretation or thoughts about the experiences, and focusing on pure present-centred attention and awareness itself (Germer 2005).

The third and final theme identified within this dimension of mindfulness was that, with presentcentred attention and awareness, Lia came across enhanced sensory experiences. These included the examples of 'mindful eating', 'mindful breathing' and 'mindful body awareness'.

'I don't really pay attention to the taste at school, just tastes like, 'Oh it's sweet', and I like chocolate so I eat it, but now I can taste, I can actually hear myself eating, I could hear the crunch of the rice crispies, the coconut and again the sweetness of the chocolate ... It was more, much more intense than when I ate this before, I think I enjoyed it now best, more than ever I guess, because I actually got to taste, no really taste it!'

As Lia's comment demonstrates, it follows then, that with full attention and awareness, one would become aware of enhanced sensory experiences as they occur in the present moment. Siegel (2007) explains that being awake to our senses results in direct contact with our moment-tomoment experiences. Similarly, Langer (2000) describes mindfulness as being actively engaged in the present, noticing new things, and being sensitive to context. The above example illustrates this aspect of Lia's mindful eating experience. Mindfulness entails being fully engaged in the present; it is a form of enhanced attention to and awareness of current experience or present reality (Brown and Ryan 2003). It is evident from the study that Lia had experiences congruent to those described and expected from the theoretical perspectives on mindfulness, regarding a heightened sensory experience resulting from full attention and awareness in the present.

It appears there is a relationship between these themes that would further enhance our understanding of Lia's subjective experience of the 'present-centred attention and awareness' dimension of mindfulness. Task-orientated attention led to enhanced sensory experiences for Lia. It also meant that Lia possibly became more aware of external happenings that distracted her attention from the task.

\section{Primary theme 2: Attitude and heart qualities}

The 'attitude and heart qualities' dimension comprises two aspects that form part of the attitude with which one practices mindfulness; firstly, intention, and secondly, the heart qualities such as compassion, non-judgement, acceptance and kindness. Bishop et al. (2004) point out that intention is a fundamental component of mindfulness that has often been overlooked in the theoretical literature. According to them, intention is the goal or personal vision that one brings to one's mindfulness practice. Furthermore, Shapiro and Schwartz (2000) maintain that mindfulness necessitates an intention to incorporate qualities such as gratitude, gentleness, generosity, empathy and lovingkindness into one's practice, and to evoke them in one's conscious attention and awareness. 
In Lia's experience of this dimension of mindfulness, the theme of understanding mindfulness emerged, that is, as her awareness of what mindfulness is increased, so too did her intention to be mindful. Again, Lia's intention was related to staying task-oriented, as that was a predominant feature of her subjective experiences of mindfulness.

'The first session, your phone rang and my attention like immediately went there, but now I can hear the children playing but l'm not focusing on them. (So what are you saying to yourself about that?)... "Focus on what you're doing," and like l'm not letting any distractions in while I'm talking to you, although I can hear them around me.'

This example illustrates Lia's goal in terms of her own personal understanding of mindfulness, which was to keep her attention focused on our conversation. It also ties in to the theme of being task-oriented. Lia perceived mindfulness as maintaining her present-centred attention on the identified task. As she realised and understood this aspect of mindfulness, her intention to remain task-oriented increased.

In light of the heart qualities of mindfulness practice, Lia also appeared to experience movement from a sense of perfectionism towards 'letting go' of the negative elements of perfectionism such as self-judgement and criticism. Lia initially strived to practice mindfulness to the best of her ability and was fairly harsh on herself when she perceived that she was not doing so. She did, however, appear to realise that she could adopt a more compassionate attitude towards herself as the study progressed, thereby letting go of some perfectionism and experiencing more of the heart qualities associated with mindfulness practice.

'... but sometimes I'm like quite harsh with myself but then I'm like, 'I don't have to do this, you know, I could just bring my mind back, I don't have to tell myself to do it." "

It appeared that during the study, Lia's self-criticism almost acted as a barrier preventing her from fully engaging in mindfulness. Her attention and awareness seemed to be frequently interrupted by her inner 'voice', which hampered her full involvement in the process. This theme also links to the notion of 'reflexive consciousness' (Brown and Ryan 2003). Lia's thinking about her thinking and interpretation of her experiences interfered with a full experience of mindfulness. On the other hand, it could be that part of this process was her attempt to reach a better state of mindfulness, which makes sense in terms of her own definition of what it means to be perfectionist. Even so, within the 'attitude and heart qualities' dimension of mindfulness, it seemed that Lia experienced difficulty embracing the ideals of compassion, gentleness and non-judgement towards herself for a large part of the study. This theme is dynamic, however, and, as mentioned, Lia appeared to make an attitudinal shift when she realised that she could 'let go' of the self-criticism and judgemental aspect of perfectionism. Thus, further on in the study, she appeared to begin to embrace the idea of compassion and non-judgement towards herself, thereby experiencing more of the heart qualities so fundamental to true mindfulness practice.

\section{Primary theme 3: Self-regulation}

Within the context of mindfulness, self-regulation is referred to as it applies to an individual's regulation of his or her attention and awareness. Thus, for the purpose of the study, self-regulation was defined as the conscious, intentional effort to control one's attention and awareness. Stability and continuity of this awareness and attention requires self-regulation (Bishop et al. 2004). Furthermore, Brown and Ryan (2003) indicate that mindfulness is linked to heightened self-knowledge, which is a key element of self-regulation, and provides evidence for the self-awareness aspect of self-regulation.

Mindfulness may help to disengage people from automatic thoughts and unhealthy behaviour patterns, and in so doing, facilitate self-regulation, which is linked to the enhancement of well-being (Ryan and Deci 2000 as cited in Brown and Ryan 2003, Shapiro and Schwartz 2000). Four themes of Lia's subjective experiences of mindfulness emerged from the data in light of the 'self-regulation' of attention and awareness as it applies to mindfulness practice, which will now be reviewed. 
Firstly, it became clear that Lia's self-regulation of attention in general is interest-driven. This applied to her mindfulness practice as well; as her interest in mindfulness developed, as did her ability to self-regulate her attention and awareness in mindfulness.

'I think being so interested in it (mindfulness) made me want to learn it faster and apply it as well.'

In general, it appears that current literature does not refer to an interest component to selfregulation of attention and awareness in mindfulness. Perhaps this is because ideal mindfulness practice does not allow one to 'choose' what to be mindful of. Self-regulation is simply the cognitive process that allows one to regulate attention and awareness to whatever internal and external experiences are unfolding each moment, regardless of whether they are desirable or not. It is also possible that by 'interest', Lia was referring to her degree of motivation to regulate her attention. In this case, she would be more motivated to self-regulate her attention if she were interested in the thing to direct her attention towards.

Secondly, Lia experienced the need for silence in order to effectively self-regulate her attention in particular.

II was just like hoping they would stop I guess, because I found it not hard, but harder to listen to what he was saying and focus completely on that and as well as breathing. So yes, I was just wanting to the noises to stop.'

Mindfulness literature does not appear to specify clearly that the practice of mindfulness necessitate silence. It might, however, be that if one is engaging in mindfulness meditation, silence will assist in the focusing of attention and awareness in the here and now. In response to his own question - 'Why silence?' - Siegel (2007: 72) notes that silence creates a 'rare opportunity to pause and drop into stillness, to become intimate with your own mind'. Becoming 'intimate' with one's mind is one of the goals of mindfulness; that is, to allow the contents of our minds to surface and flow freely; to become the observer. Siegel (2007) thus implies that silence facilitates the achievement of this objective. He further explains that stillness is not a lack of activity, but rather a 'stabilizing strength' that allows us to observe the transient nature of our mind as opposed to the apparent permanent nature of thoughts and feelings (Siegel 2007: 73). Silence opens the doors for us to enter into a place of more mindfulness.

It is our interpretation that, being the 'perfectionist' that she is, by referring to the need for silence Lia also implied her attempts to reach a 'better' state of mindfulness. Lia confirmed this interpretation, explaining that having silence helped her apply effort to 'getting it right'. Lia's aim to attain a more mindful state adds another element to the theme of needing silence in order to effectively selfregulate her attention in mindfulness practice. Furthermore, it is linked to her experiences within both the 'present-centred attention and awareness' and 'attitude and heart qualities' dimensions of mindfulness, as discussed above. On the other hand, there were times during this study when the use of sound actually assisted Lia to self-regulate her attention. For example, the use of Kabat-Zinn's guided meditations on CD seemed to help Lia to achieve some degree of mindfulness. Nairn (1998) describes a type of meditation where one uses sound to actually support one's focus of attention during the meditation - almost as an 'anchor'. He explains that in this case, special sounds such as music are not created, but that one should allow surrounding sounds of the moment to provide the focus - the sound of 'traffic, wind, voices ... whatever is there will do' (Nairn 1998: 25).

The third theme that emerged relating to this dimension was that it required substantial effort for Lia to self-regulate her attention and awareness in mindfulness.

'... mostly trying to recognise what it is, but I don't really know, l'm trying to focus on it ...'

Once again, it seems that Lia's efforts to self-regulate her attention in the mindfulness activities had the aim of being able to achieve a better state of mindfulness. Nonetheless, the idea of effort seemed to be a dominant theme during the study, and Lia confirmed these interpretations 
during member-checking. This finding came as no surprise; and the literature does not state that mindfulness is an easy practice! If anything, much of the literature describes the great challenges of attaining mindfulness.

Mindfulness is deceptive in that it appears outwardly simple and straightforward, yet an in-depth analysis reveals the intricacies and multiple layers of this challenging phenomenon. Siegel (2007: 55) describes his personal experience of trying to maintain a mindfulness state during a meditation retreat: 'After a few moments it seems I can barely make it through an entire breath without having my mind pulled toward different thoughts like a dog zig-zagging on a walk, drawn this way and that by enticing scents along the path.' Nairn (1998: 22) confirms that, 'the average human mind is in a constant state of distraction' and he describes the arising difficulties in mindfulness and meditation, due to this nature of the mind. Kabat-Zinn (2003: 148) also emphasises the overall complexity of mindfulness practice: 'Mindfulness is not merely a good idea such that, upon hearing about it, one can immediately decide to live in the present moment, with the promise of reduced anxiety and depression and heightened performance and life satisfaction, and then instantly and reliably realise that state of being.' He compares it to an art form and way of being that can only be developed through ongoing effort and practice over time, stating that 'it takes personal commitment and perseverance in formal practice gradually to establish a degree of stability in one's capacity to attend ...' (Kabat-Zinn 2003: 150).

Similarly then, it seems that self-regulation is by no means a simple task, especially for Lia. Bishop et al. (2004) maintain that the metacognitive skill of mindfulness can be developed with practice, and the aim of Gestalt therapy is to facilitate the development of clients' self-regulation of attention to be fully present in the now. The literature thus suggests that mindfulness, and particularly the self-regulation component of mindfulness, is a skill that can be developed. This implies that it is not simple and straightforward, as Lia experienced, but that it can be improved upon with facilitation and practice.

The data indicated that Lia did experience this developmental aspect of her self-regulation skills, as an additional theme is that they improved as her awareness increased.

'Well l've realised what it actually is, so I guess like the better, the more sessions we have the better understanding I have of it and the more I can practice it.'

This theme correlates with what the literature suggests - i.e. that the metacognitive skill of mindfulness can be developed with practice, and that greater awareness and exposure contributes towards improved quality of mindfulness (Bishop et al. 2004, Kabat-Zinn 2003, Nairn 1998).

\section{Secondary theme 1: Mindlessness}

Mindlessness is the 'unawareness' of our habitual and automatic behaviours (Thompson and Gauntlett-Gilbert 2008, Brown and Ryan 2003). By becoming aware of our mindlessness in various situations, we are able to fine-tune our mindfulness practice. As Lia experienced mindfulness through exposure and practice, she realised what mindlessness is, which in turn enhanced her mindfulness practice. This relationship appeared to apply conversely as well. Thus the theme identified within this dimension of mindfulness was an increased awareness of what mindlessness is.

'Today I wasn't very mindful, I just wanted to eat because I was so hungry, so I just ate and I was watching TV ... But yesterday I tried to be mindful and I ate very little, surprisingly ... Usually I just stuff myself, like eating would be over ... Usually I just eat to get full!'

This theme illustrates that Lia experienced an increase in her awareness of when she engages in mindless as opposed to mindful behaviour. This particular experience confirms what the literature describes about mindlessness. For instance, Brown and Ryan (2003) include being occupied with multiple tasks, and compulsive and automatic behaviours, as examples of mindlessness. In the above example, Lia realised that she was behaving mindlessly when she was eating in front of the television (multiple tasks), as well as when she was engaging in the automatic behaviour of "just 
eating to get full'. Lia thus seemed to realise the application of mindfulness and mindlessness in her everyday life. By examining mindlessness and how it potentially affects our well-being, we gain a greater understanding of mindfulness, and its role in mental health and general well-being. Thus mindlessness is not a dimension of the mindfulness experience per se; but its addition contributes to a more in-depth understanding of mindfulness.

\section{Secondary theme 2: Universalism of mindfulness}

The phenomenon of mindfulness often appears to be linked to the idea of 'meditation', possibly due to its origins in the 'mindfulness meditation' of Buddhist traditions (Kabat-Zinn 2003). In light of this, it is important to differentiate between mindfulness and meditation. Mindfulness by its nature is essentially a universal phenomenon, thus it is not affiliated to any particular religion. It has been defined as a psychological process, which can be enhanced or achieved by meditation, as well as various other methods that have nothing at all to do with meditation. Brown and Ryan (2003) maintain that almost everyone has the capacity to attend and to be aware, but that the capacity as well as willingness varies amongst individuals. Mindfulness is thus seen as more than meditation; meditation is simply a scaffolding technique employed to develop the state and skills of mindfulness (Kabat-Zinn 2005).

The first theme within this dimension was the conflict that Lia experienced in the initial stages of the study regarding mindfulness' place in science and religion. The theme of her uncertainty as to where mindfulness is located within these two contexts was apparent.

'He spoke about, I think, the mind being a sixth sense, that also stood out a lot ... He said that, ... okay back to my dad, he believes strongly in science, okay, and Jon spoke about scientists, as well as about like Buddhists I think, discovering that the mind is a sixth sense, so yeah, seeing it from both views stood out a lot.'

The conflict Lia appeared to experience in this regard stemmed from a past experience. She had once attended a religious ceremony where she witnessed somebody in a 'trance-like' state, which was traumatic for her. Her father had explained that it was a strong form of meditation that had enabled that person to accept that 'state of mind'. Lia explained that her family is not very religious, and her father in particular believes in science over religion. When listening to the $C D$ and hearing Kabat-Zinn explain mindfulness as a 'state of mind', Lia associated it with her past experience.

Almost every human being is able to attend and be aware (i.e. be mindful), although the degrees of awareness will vary between individuals (Brown and Ryan 2003, Kabat-Zinn 2003). Mindfulness is therefore an event that occurs naturally in everyday life, although it requires practice to be maintained (Germer 2005). Germer (2005: 16) describes mindfulness as 'settling into our current experience in a relaxed, alert and openhearted way'. This aspect of universalism in mindfulness thus implies that it is something that can be cultivated in everyday life. A similar theme of mindfulness being a way of life emerged in terms of Lia's subjective experiences of this mindfulness dimension, during the latter part of the study. She seemed to interpret mindfulness as a technique that she could directly apply to her everyday life.

'I learn a lot every time and it's quite hard to explain it in words ... so I know now it's a way of life ... because I'm applying it every day and I think that it could help whoever, whenever, wherever.'

As mentioned, Germer (2005) maintains that moments of mindfulness occur naturally in everyday life, and Kabat-Zinn (2003) explains that we are all mindful in varying degrees, each moment of our lives. Although Lia's experience does echo an aspect of the literature in that she realised that mindfulness can be applicable to daily life, there is a difference in this theme compared to what the literature suggests. That is, Lia seemed to interpret that mindfulness is a technique in itself that can be directly applied by herself to her life. There is a sense of agency within this theme. What the literature indicates, on the other hand, is that mindfulness exists naturally within daily life already; 
as something to be developed from a natural occurrence. The literature emphasises that due to the nature of mindfulness as involving consciousness, it exists universally because we are conscious beings. It is therefore something to be recognised, practised and developed in our daily lives. In contrast, Lia's comments suggest that she viewed mindfulness as a technique to be mastered and then applied. She did not seem to think of it as something already in existence.

\section{Overall synthesis of findings}

In summary, an overall analysis of the results and findings of the study suggests two prevailing conclusions that come to the fore in terms of Lia's subjective experiences of mindfulness: (1) being task-oriented, and (2) experiencing personal growth and development.

Firstly, Lia's subjective experiences of mindfulness appear to have been predominantly task-oriented overall. The examination of the findings of this study reveal that, although task-orientation emerged as a strong theme within the 'present-centred attention and awareness' dimension of Lia's subjective experiences of mindfulness, it was linked to all the dimensions. Being task-oriented in her mindfulness practice was also evident in Lia's subjective experiences of her intention to be mindful, as well as the 'perfectionism' that she applied to all identified tasks - both aspects of the 'attitude and heart qualities' dimension. Furthermore, task-orientation was also tied in to Lia's subjective experiences of her self-regulation of attention as applied to mindfulness practice. Task-orientation ties in to mindlessness in that an awareness of mindlessness assisted Lia to become more mindful and to refocus her attention on identified tasks. In terms of the universalism of mindfulness, being task-orientated applied to tasks in everyday life for Lia. The dimensions of mindfulness identified from the literature were presented individually, but it is imperative to view them as interconnected. They all interact dynamically to create the complete experience of mindfulness. It therefore follows that this conclusion of Lia's subjective experiences of mindfulness being task-oriented overall should be evident across the dimensions.

This same principle applies to the second conclusion that can be drawn from the results of the study. The theme of Lia experiencing personal growth and development through her mindfulness practice emerges across almost all the identified dimensions of mindfulness. As discussed, Lia experienced development within the 'attitude and heart qualities' dimension in that she moved from an initial perfectionism towards letting go of the associated negative aspects. As Lia's understanding and experience of mindfulness increased, her ability to self-regulate her attention in mindfulness also appeared to develop, as well as her awareness of mindlessness and the universalism of mindfulness. Lia thus appeared to experience personal growth and development in her understanding and practice of mindfulness, in terms of most aspects of the framework within which her experiences were interpreted. It seems that, overall, as Lia's awareness of what mindfulness entails and her experiences thereof increased during the study, so she developed personally, specifically in terms of her 'skills' (maintaining present-centred attention and awareness, selfregulating attention and awareness, and adopting heart qualities) in mindfulness practice.

\section{Concluding remarks}

Several factors that are potential limitations of the study were identified. Firstly, due to the nature of the study and the data creation methods applied, a close working relationship was developed with Lia. As participant-observer, the first author was directly involved during each session, and was therefore susceptible to the possibility of researcher-induced bias. As researcher in this study the author became vulnerable to the possibility of subjectivity in her observations and interpretations, and had to maintain a professional approach to the research at all times, setting clear boundaries about her role. Keeping a research diary, reflecting on her experiences in this regard, and briefings with her supervisor assisted in preventing subjectivity as far as possible.

Secondly, the nature of the research topic was quite complex, resulting in some explanations of mindfulness during discussions with Lia. These discussions therefore included some of the author's 
own interpretations and experiences of mindfulness, which may have influenced Lia's understandings and sharing of her experiences. This is a limitation from one perspective, however on the other hand; the sharing discussions were advantageous from another angle. They enabled the building of trust and an open relationship between Lia and the researcher, which became a 'safe' space for her to be honest and to share as much of her experiences as possible. This in turn facilitated the rich descriptions and in-depth understandings sought from the study.

A further potential limitation of the study relates to Lia's style of interaction with her world. She openly described herself as a 'perfectionist', wanting to do everything to the best of her ability in order to satisfy herself and to impress her best upon others. It is possible that she could have discussed her subjective experiences of mindfulness in terms of what she perceived the best experiences to be. It is also possible that she wanted to 'please' the researcher. Although this may have been a limitation in some parts of the study, a careful examination of the data leaves the impression that this was not the case overall.

A delimitation of the study is that it is restricted to a single case study; therefore the findings are not necessarily generalisable. The research must be read with this in mind; but it is also important to note that this was not the purpose of the study and a specific design choice. This study particularly focused on a single case in order to generate the rich descriptions and deep understandings sought from an interpretivist paradigm.

The rich descriptions and in-depth understandings generated from this study serve as a potential contribution to be made to understand an adolescent's subjective experiences of mindfulness. It has been made clear that a substantial gap in the literature exists from this perspective. There is a lack of qualitative investigation on the topic of mindfulness, as well as a lack of research focusing on mindfulness as it potentially pertains to children and adolescents. This study could contribute to the identified gap in the available literature. Mindfulness has been identified as holding much promise as a positive phenomenon that can be developed in the maintenance of physical, mental and emotional health and well-being. This is especially relevant in light of positive psychology and trends towards more holistic approaches to health and well-being.

Therefore, mindfulness shows potential promise as a positive phenomenon that can be cultivated in the development of health and well-being. It is recommended that practitioners in the health professions and in education consider mindfulness as a potentially useful technique, particularly with the youth. Given the complexity of mindfulness as discussed, practitioners need to receive adequate training in the conceptualisation and applications of mindfulness. With the appropriate training and foundations, however, practitioners in a wide variety of contexts can apply mindfulness, within their scope of practice. Furthermore, it is preferable that practitioners engage in their own personal mindfulness practice, in order to effectively apply it in their professional practice.

In the application of mindfulness in practice, professionals are also encouraged to familiarise themselves with the specific moderations that can be made to mindfulness for children and adolescents. Thompson and Gauntlett-Gilbert (2008) explain that this specific population group require greater explanation and rationale, use of different practices, useful metaphors, variety and repetition, shorter practice times, involving parents, and mindfulness in groups.

Recommendations for further research include broadening the scope and engaging in largerscale studies, conducting studies with younger children, utilising different research designs, and exploring the effectiveness of mindfulness as an intervention technique in addressing scholastic, social, behavioural and/or emotional difficulties in children and adolescents.

In summary, in the words of Siegel (2007: 15): 'We are in desperate need of a new way of being - in ourselves, in our schools, and in our society. Our modern culture has evolved in recent times to create a troubled world with individuals suffering from alienation, schools failing to inspire and to connect with students, in short, society without a moral compass to help clarify how we can move forward in our global community.' It is hoped that this article has provided insight into the phenomenon of mindfulness, how an adolescent subjectively experienced it, and how it might be able to play a part in the positive transformation of the global community, in the context of a move towards greater holistic health and well-being. 


\section{References}

Baer RA (2003) Mindfulness training as a clinical intervention: a conceptual and empirical review. Clinical Psychology: Science and Practice 10(2): 125-143

Bishop SR, Lau M, Shapiro S, Carlson L, Anderson ND and Carmody J (2004) Mindfulness: a proposed operational definition. Clinical Psychology: Science and Practice 11(3): 230-241

Bootzin RR and Stevens SJ (2005) Adolescents, substance abuse, and the treatment of insomnia and daytime sleepiness. Clinical Psychology Review 25: 629-644

Brown KW and Ryan RM (2003) The benefits of being present: mindfulness and its role in psychological well-being. Journal of Personality and Social Psychology 84(4): 822-848

Brown KW, Ryan RM and Creswell JD (2007) Mindfulness: theoretical foundations and evidence for its salutary effects. Psychological Inquiry 18(4): 211-237

Coetzee S and Viviers R (2007) An overview of research on positive psychology in South Africa. South African Journal of Psychology 37(3): 470-490

Creswell JW (2002) Educational Research: Planning, Conducting, and Evaluating Quantitative and Qualitative Research. Upper Saddle River, NJ: Merrill Prentice Hall

Denzin NK and Lincoln YS (eds) (2003) Collecting and Interpreting Qualitative Materials (2nd edn). Thousand Oaks, CA: Sage Publications

De Vos AS, Strydom H, Fouche CB and Delport CSL (2005) Research at Grass Roots for the Social Sciences and Human Service Professions (3rd edn). Pretoria: Van Schaik

Germer CK (2005) Mindfulness: What is it? What does it Matter? In: Germer CK, Siegel RD and Fulton PR (eds), Mindfulness and Psychotherapy. New York: The Guilford Press. pp 3-54

Hatch JA (2002) Doing Qualitative Research in Education Settings. New York: State University of New York Press

Kabat-Zinn J (2003) Mindfulness-based interventions in context: Past, present, and future. Clinical Psychology: Science and Practice 10(2): 144-156

Kabat-Zinn J (2005) Coming to Our Senses: Healing Ourselves and the World through Mindfulness. New York: Hyperion

Kabat-Zinn J (2006) Mindfulness for Beginners. Louisville, CO: Sounds True

Langer EJ (2000) Mindful learning. Current Directions in Psychological Science 9(6): 220-223

Lincoln YS and Guba EG (1985) Naturalistic Inquiry. Thousand Oaks, CA: Sage Publications

Matchim Y and Armer JM (2007) Measuring the psychological impact of mindfulness meditation on health among patients with cancer: a literature review. Oncology Nursing Forum 34(5): 1059-1066

Nairn R (1998) Diamond Mind: Psychology of Meditation. Cape Town: Kairon Press

Seligman MEP and Csikszentmihalyi M (2000) Positive psychology: an introduction. The American Psychologist 55(1): 5-14

Semple RJ, Reid EFG and Miller L (2005) Treating anxiety with mindfulness: an open trial of mindfulness training for anxious children. Journal of Cognitive Psychotherapy 19(4): 379-392

Shapiro SL and Schwartz GER (2000) Intentional systemic mindfulness: an integrative model for self-regulation and health. Advances in Mind-Body Medicine 16(2): 128-134

Siegel DJ (2007) The Mindful Brain: Reflection and Attunement in the Cultivation of Well-Being. New York: WW Norton and Company

Singh NN, Lancioni GE, Singh Joy SD, Winton ASW, Sabraawi M and Wahler RG (2007) Adolescents with conduct disorder can be mindful of their aggresive behaviour. Journal of Emotional and Behavioural Disorders 15(1): 56-63

Stake RE (2005) Qualitative case studies. In: Denzin NK and Lincoln YS (eds), The Sage Handbook of Qualitative Research (3rd edn). Thousand Oaks, CA: Sage Publications. pp 443-466

Thompson M and Gauntlett-Gilbert J (2008) Mindfulness with children and adolescents: effective clinical application. Clinical Child Psychology and Psychiatry 13(3): 395-407

Wall RB (2005) Tai Chi and mindfulness-based stress reduction in a Boston public middle school. Journal of Pediatric Health Care 19(4): 230-237

Yin RK (2003) Case Study Research: Design and Methods (3rd edn). Thousand Oaks, CA: Sage Publications 